NBI-HE-93-3

January 1993

\title{
4d quantum gravity coupled to matter
}

\author{
J. Ambjørn \\ The Niels Bohr Institute \\ Blegdamsvej 17, DK-2100 Copenhagen Ø, Denmark \\ Z. Burda ${ }^{1}$ and J. Jurkiewicd \\ Inst. of Phys., Jagellonian University., \\ ul. Reymonta 4, PL-30 059, Kraków 16, Poland \\ C.F. Kristjansen \\ The Niels Bohr Institute \\ Blegdamsvej 17, DK-2100 Copenhagen Ø, Denmark
}

\begin{abstract}
We investigate the phase structure of four-dimensional quantum gravity coupled to Ising spins or Gaussian scalar fields by means of numerical simulations. The quantum gravity part is modelled by the summation over random simplicial manifolds, and the matter fields are located in the center of the 4-simplices, which constitute the building blocks of the manifolds. We find that the coupling between spin and geometry is weak away from the critical point of the Ising model. At the critical point there is clear coupling, which qualitatively agrees with that of gaussian fields coupled to gravity. In the case of pure gravity a transition between a phase with highly connected geometry and a phase with very "dilute" geometry has been observed earlier. The nature of this transition seems unaltered when matter fields are included. It was the hope that continuum physics could be extracted at the transition between the two types of geometries. The coupling to matter fields, at least in the form discussed in this paper, seems not to improve the scaling of the curvature at the transition point.
\end{abstract}

\footnotetext{
${ }^{1}$ Supported by the KBN grants no. 200539101 and 2P302-16904
} 


\section{Introduction}

Last year a new regularized model of quantum gravity in 4D was introduced [1, 2]. The path integral is approximated by a summation over randomly triangulated piecewise linear manifolds. This method is a generalization of the one from two dimensions, which was very successful [4, 5, 7, 6. In 4D simplicial quantum gravity two different phases have been observed, one with a highly connected geometry and a large Hausdorff dimension and one with a low Hausdorff dimension. Based on numerical simulations it was suggested in [2] that the transition between the two types of geometries was of second order and that an interesting continuum limit might be extracted at the transition point. This observation has been further corroborated in a sequence of papers [8, 9, 10, 11, 12.

One obstacle to the above mentioned suggestion is that the average curvature does not scale to zero at the transition point. The average curvature does decrease (albeit slowly) with the volume of the simulated universes and it cannot be completely ruled out that it actually scales to zero in the infinite volume limit. However, at the moment we consider it as unlikely. This prompts at least a reinterpretation of the meaning of the scaling limit since naive scaling like

$$
\left\langle R_{\text {lattice }}\right\rangle=\left\langle R_{\text {cont }}\right\rangle a^{2}
$$

(where $a$ is the lattice spacing) cannot be maintained. Maybe the average curvature should be absorbed in a redefinition of the cosmological constant, while the relevant physical curvature arises only through fluctuations around the "fictitious" average curvature. While such an unconventional limit might exist, it seems not to be very natural to us. An attempt to improve the situation by adding terms like $R^{2}$ to the action was not very successful [10]. At this point we should mention a recent suggestion 13] of a different identification of the lattice results with continuum theory in which one considers the limit of the bare gravitational coupling constant going to infinity. This limit might in continuum language correspond to an infrared fixed point dominated by the quantum fluctuations of the conformal factor. The scaling relations derived in [13] agree at the qualitative level quite well with the numerical results, but they move the interesting region of continuum physics away from the transition in geometry and to a region in coupling constant space where (1]) can be satisfied. We consider this suggestion as most interesting. In this article we explore

\footnotetext{
${ }^{1}$ An older, related approach makes use of a fixed triangulation, but allows the variation of the length of the links. Contrary, in the present approach one keeps the length of the links fixed, but varies the connectivity. We refer to [3] for a recent lucid review of the first approach, which we here will call "Regge gravity", while we will use the term "simplicial gravity" for the present approach.
} 
another way to cure the problem with the scaling of the average curvature, namely coupling of matter fields to gravity. It is of course also of interest in itself to study matter fields coupled to dynamical random geometries. In the best of all worlds one could even hope that the quest for correct scaling of gravity observables like the average curvature would uniquely determine the matter content of the theoryt?

The coupling of matter to two-dimensional gravity has revealed a rich and beautiful structure as long as the central charge of the field theory is less than or equal to one. This is summarized in the KPZ formulas [14, but was first discovered in the simplicial gravity approach. As an example, when the Ising model is coupled to $2 \mathrm{~d}$ simplicial gravity its phase transition changes from being second order to third order [15, 16]. In addition the back-reaction of matter changes the critical exponent $\gamma$ of gravity at the critical point of the Ising model. Away from the critical point this exponent is unchanged.

Unfortunately the analytical methods of 2d have not yet been extended to higher dimensions. The coupling of the Ising model to $3 \mathrm{~d}$ gravity was investigated by numerical simulations in [17, 18, 19]. The phase diagram was determined in [19] and the conclusion was that, although there was a clear coupling between gravity and the spins at the critical point of the spin system, this influence was not sufficiently strong to change the first order transition observed in three dimensions [20, 21] between the two phases of the geometrical system into a more interesting (from the point of view of continuum physics) second order transition. In this respect the situation is better in $4 \mathrm{~d}$ where the transition between the two phases of the geometrical system may already be of second order, as mentioned above.

The rest of this paper is organized as follows: In section 2 we define the model. In section 3 we discuss briefly the numerical method, while section 1 contains our numerical results. Finally in section 5 we discuss the results obtained.

\section{The model}

Simplicial quantum gravity in $4 \mathrm{~d}$ is described by the following partition function (see e.g. [2, 10]):

$$
Z\left(\kappa_{2}, \kappa_{4}\right)=\sum_{T \in \mathcal{T}} e^{-\kappa_{4} N_{4}+\kappa_{2} N_{2}}
$$

where the sum is over triangulations $T$ in a suitable class of triangulations $\mathcal{T}$. The quantity $N_{4}$ denotes the number of 4-simplexes in the triangulation and $N_{2}$ the

\footnotetext{
${ }^{2}$ But we will of course not seriously pretend, that the present stage of numerical simulations of quantum gravity is such, that one could really determine the matter content.
} 
number of triangles. The coupling constant $\kappa_{2}$ is inversely proportional to the bare gravitational coupling constant, while $\kappa_{4}$ is related to the bare cosmological constant. The most important restriction to be imposed on $\mathcal{T}$ is that of a fixed topology. If we allow an unrestricted summation over all topologies in (2) the partition function is divergent [2]. In the following we will always restrict ourself to consider manifolds with the topology of $S^{4}$.

$Z\left(\kappa_{2}, \kappa_{4}\right)$ is the grand canonical partition function. It is defined in a region $\kappa_{4} \geq \kappa_{4}^{c}\left(\kappa_{2}\right)$ in the $\left(\kappa_{2}, \kappa_{4}\right)$ coupling constant plane. The only way in which we can hope to obtain a continuum limit is by letting $\kappa_{4}$ approach $\kappa_{4}^{c}\left(\kappa_{2}\right)$ from above. This tentative continuum limit depends only on one coupling constant $\kappa_{2}$ and the transition between the two phases of $4 \mathrm{~d}$ gravity mentioned above takes place at a critical value of $\kappa_{2}, \kappa_{2}^{c}$. It is often convenient to think about the canonical partition function where $N_{4}$ is kept fixed. Then $\kappa_{2}$ is the only coupling constant and the aspects of gravity which do not involve the fluctuation of the total volume of the universe can be addressed in the limit of large $N_{4}$. For the geometrical system an observable which has our interest is the average curvature per volume, $\langle R\rangle$. The average curvature can for a simplicial manifold be defined by Regge calculus and in the case of equilateral simplexes one simply has

$$
\langle R\rangle \propto\left(c_{4} N_{2} / N_{4}-10\right)
$$

where the constant $c_{4}$ is the number of 4-simplexes to which each triangle should belong if the manifold were flat. Furthermore one can by an appropriate interpretation of the Regge approach introduce the average of the squared curvature per volume by

$$
\left\langle R^{2}\right\rangle \propto \frac{\sum_{n_{2}} o\left(n_{2}\right)\left[\left(c_{4}-o\left(n_{2}\right)\right) / o\left(n_{2}\right)\right]^{2}}{10 N_{4}}
$$

where the sum is over triangles $n_{2}$ and $o\left(n_{2}\right)$ is the order of a given triangle i. e. the number of 4 -simplexes to which this triangle belongs. The correlator $\left\langle R^{2}\right\rangle-\langle R\rangle^{2}$ will prove useful as an indicator of a change in geometry.

One can now couple matter fields to simplicial quantum gravity. In the case of Ising spins the partition function will look like:

$$
Z\left(\beta, \kappa_{2}, \kappa_{4}\right)=\sum_{N_{4}} e^{-\kappa_{4} N_{4}} \sum_{T \in \mathcal{T}\left(N_{4}\right)} \sum_{\{\sigma\}} e^{\kappa_{2} N_{2}} e^{\beta \sum_{\langle i, j\rangle}\left(\delta_{\sigma_{i} \sigma_{i}}-1\right)} .
$$

In this formula $\mathcal{T}\left(N_{4}\right)$ signifies the subclass of $\mathcal{T}$ with volume $N_{4}, \sum_{\{\sigma\}}$ the summation over all spin configurations, while $\sum_{\langle i, j\rangle}$ stands for the summation over all neighbouring pairs of 4 -simplexes. As a function of $\beta$ there might or might not be 
a phase transition for the spin system, depending on the value of $\kappa_{2}$ (assuming that $\kappa_{4}=\kappa_{4}^{c}\left(\kappa_{2}, \beta\right)$, where $\kappa_{4}^{c}$ now depends on both $\kappa_{2}$ and $\beta$ ).

The coupling of scalar fields to simplicial quantum gravity is also straightforward. Here we will ignore self-interaction of the scalar fields and direct coupling between the scalar fields and the curvature, and simply consider the following partition function

$$
Z\left(\kappa_{2}, \kappa_{4}\right)=\sum_{N_{4}} \sum_{T \in \mathcal{T}\left(N_{4}\right)} e^{\kappa_{2} N_{2}-\kappa_{4} N_{4}} \int \prod_{i, \alpha} \frac{d \phi_{i}^{\alpha}}{\sqrt{2 \pi}} \prod_{\alpha=1}^{n_{g}} \delta\left(\sum_{i} \phi_{i}^{\alpha}\right) e^{-\frac{1}{2} \sum_{\langle i, j\rangle, \alpha}\left(\phi_{i}^{\alpha}-\phi_{j}^{\alpha}\right)^{2}} .
$$

Here $i$ labels the 4-simplexes, $\alpha$ different components of the field $\phi$ and $n_{g}$ is the total number of independent Gaussian fields. There is no need for a coupling constant in front of the Gaussian action since it can always be absorbed in $\kappa_{4}$ by a rescaling of the $\phi$ 's. Of course the gaussian action can in principle be integrated out explicitly, leaving us with an additional weight

$$
\left(\operatorname{Det} C_{T}\right)^{-n_{g} / 2}
$$

for each triangulation $T$, where $C_{T}$ is just the incidence matrix for the $\varphi^{5}$-graph which is dual to the triangulation $T$. In the case of gaussian fields coupled to $2 \mathrm{~d}$ gravity this fact was used to determine qualitatively the phase diagram of noncritical strings as a function of the number of Gaussian systems, $n_{g}$ 22, 23, 24]. In principle one could try to do the same here. However, the class of allowed $\varphi^{5}$ graphs is not so easy to determine as in the case of $2 \mathrm{~d}$ gravity. In the following we will rely on numerical simulations.

\section{$3 \quad$ Numerical methods}

One annoying aspect of the above formalism is that we are forced to perform a grand canonical simulation where $N_{4}$ is not fixed. The reason is that we have no ergodic updating algorithm ${ }^{3}$ which preserves the volume $N_{4}$. It is however possible to perform a grand canonical updating without violating ergodicity and still stay in the neighbourhood of a prescribed value of $N_{4}$, which we will denote $N_{4}(f i x)$. The procedure involves finetuning of $\kappa_{4}$ to its critical value, $\kappa_{4}^{c}\left(\kappa_{2}, \beta\right)$. We refer to 10 for details.

In addition to the updating of the geometry, we also have to update the Ising spin system and the Gaussian systems. Let us first discuss the Ising spin system.

\footnotetext{
${ }^{3}$ In 2 d gravity we know how to perform a canonical updating, but even there the grand canonical updating is occasionally convenient to use [25, 22, 23, 26.
} 
In order to avoid critical slowing down close to the phase transition between the magnetized and the non-magnetized phase the spin updating is performed by the single cluster variant of the Swendsen-Wang algorithm developed by Wolff [27]. The cluster updating algorithms have been successfully applied to the Ising model coupled to $2 \mathrm{~d}$ gravity [28, 29, 30, 31] and to the Ising model coupled to $3 \mathrm{~d}$ gravity [19]. We update the spins once for every sweep, i.e. after $N_{4}($ fix $)$ accepted updatings of the geometry.

In the simulations we have scanned the $\left(\kappa_{2}, \beta\right)$ coupling constant plane by first fixing $\kappa_{2}$ and then varying $\beta$ in the search for a critical value $\beta_{c}\left(\kappa_{2}\right)$ where the spin system undergoes a transitionf. For values of $\kappa_{2}$ where we are well inside the phase with a highly connected geometry where and a large Hausdorff dimension, 5000 sweeps are sufficient to achieve equilibrium for bulk quantities when the number of simplexes does not exceed $N_{4}=9000$. This is in agreement with the situation in pure gravity [2, 10]. We have occasionally made longer runs in connection with the measurement of Binders cumulant (50.000 sweeps) and near critical points either in the spin or gravity coupling constant. It seems as if the situation is in all respects as in $2 \mathrm{~d}$ and $3 \mathrm{~d}$ gravity. In particular the presence of the spins seems not to slow down the convergence of bulk geometric observables (in $2 \mathrm{~d}$ it is known that spins speed it up). In this phase we have neither seen excessive signs of autocorrelations of spins (the longest of the order of 500 sweeps at the spin transition). This is in agreement with intuition since the connectivity of the system is large and the maximal distance between spins correspondingly small. The situation is somewhat different when we probe the phase where the geometry is elongated and where internal distances can be quite large. Without spin the convergence in geometry is slow in this phase and it is true also after coupling to spins.

The Gaussian fields are updated by a heatbath algorithm. There are two aspects of this updating. One type of updating is performed with a fixed background geometry and is standard. The other one is related to the Metropolis updating of the geometrical structure. Since there are slightly unconventional aspects connected with the change of the fields, when the geometry is changed ?, let us make a few comments. We will not go into details (which are trivial, but clumsy to write down explicitly), but rather sketch the main point: Consider a change in geometry where we take a 4-simplex, remove the "interior", insert a vertex in the "empty" interior and connect

\footnotetext{
${ }^{4}$ In order that the reader could appreciate the amount of work going into this please note that we have to fine-tune $\kappa_{4}$ for each value of $\kappa_{2}$ and $\beta$.

${ }^{5}$ The same aspect is already present in the grand canonical algorithms used in $2 \mathrm{~d}$ gravity, see e.g. 25].
} 
this vertex to the five vertices of the former 4-simplex. With a proper identification of sub-simplexes we have by this procedure removed one 4-simplex and created five new ones. The inverse "move" is one where we remove a vertex of order five and the associated five 4-simplexes and replace them by a single 4-simplex. We must be careful to treat the Gaussian fields correctly in such moves. In the case where we insert a vertex we will have to introduce five new fields $\varphi_{i}, i=1, \ldots 5$. They will interact quadratically with each other, and each of them will interact with one field associated with a neighbouring 4-simplex untouched by the move. Let us denote these five fields $\phi_{i}, i=1 \ldots 5$ In addition we have removed a field associated with the original 4-simplex. We denote it by $\varphi_{0}$. It interacted with the five $\phi_{i}$ 's. The correct probability distribution of the new five $\varphi_{i}$ 's is

$$
d P_{\text {new }}\left(\varphi_{i}\right)=C_{\text {new }}\left(\phi_{i}\right) \prod_{i=1}^{5} d \varphi_{i} e^{-S_{\text {new }}\left(\varphi_{i}, \phi_{i}\right)}
$$

where the additional part of the action $S_{\text {new }}$ coming from added fields $\varphi_{i}$, determined from (6) is

$$
S_{\text {new }}\left(\varphi_{i}\right)=\frac{1}{2} \sum_{i<j}\left(\varphi_{i}-\varphi_{j}\right)^{2}+\frac{1}{2} \sum_{i}\left(\varphi_{i}-\phi_{i}\right)^{2}
$$

The factor $C\left(\phi_{i}\right)$ is a normalization factor, which contains the exponential of a quadratic form in the $\phi_{i}$ 's and its all-over scale is fixed by the requirement that $\int d P_{\text {new }}\left(\varphi_{i}\right)=1$. In a similar way the field $\varphi_{0}$ which was removed had a Gaussian probability distribution $d P_{\text {old }}\left(\varphi_{0}\right)$, just with another action

$$
S_{\text {old }}\left(\varphi_{0}\right)=\frac{1}{2} \sum_{i}\left(\varphi_{0}-\phi_{i}\right)^{2}
$$

and an appropriate normalization factor $C_{\text {old }}\left(\phi_{i}\right)$, which again contains the exponential of a Gaussian form in $\phi_{i}$ 's. Assuming that the fields $\varphi_{0}, \ldots, \varphi_{5}$ are selected according to $P_{\text {new }}$ and $P_{\text {old }}$ it is easy to enlarge the condition for detailed balance for the change in geometry to include the additional change in field content.

The geometrical moves fall in three classes (see e.g. [2] for details) of which we have described one above. A second class is one where two neighbouring 4-simplexes are removed and replaced by three new ones having in common a link (a 1-simplex), or the inverse move, where three 4-simplexes sharing a link are removed and replaced by two 4-simplexes being neighbours (i.e. sharing a 3-simplex). Finally the third class of moves is "self-dual": three 4-simplexes sharing a triangle (a 2-simplex) are replaced by three others, sharing a different triangle. In all cases one can easily write down $d P_{\text {new }}$ and $d P_{\text {old }}$ as above and incorporate these probabilities in the requirement of detailed balance needed for performing the purely geometrical move. 
The total updating is now organized in the following way: A sweep over the lattice with an updating of geometry and the above described updating of field content is followed by a number of sweeps with the geometry fixed and ordinary heatbath updating of the Gaussian fields. The actual number of such heatbath updatings for each geometrical updating is chosen so that the fastest convergence to equilibrium is achieved. For one gaussian field two heatbath updatings for each geometrical updating is usually sufficient as long as the geometry is highly connected. In the elongated phase up to 15 gaussian updatings were needed. The number of necessary updatings per sweep increases with the number of Gaussian fields. For 4 Gaussian fields 3 updatings per sweep were needed in the highly connected phase of gravity.

\section{Numerical results}

\subsection{Ising spins coupled to gravity}

Pure $4 \mathrm{~d}$ gravity has two phases and this fact is not changed by the coupling to a single Ising spin.

In the phase where the geometry is highly connected the spin system has a phase transition. In fig. 1 we show the absolute value of the magnetization

$$
|\sigma|=\frac{1}{N_{4}}\left|\sum_{i=1}^{N_{4}} \sigma_{i}\right|
$$

as a function of $\beta$ for a value of $\kappa_{2}$ for which the geometrical system is highly connected. In fig. 2 we show Binders cumulant defined by

$$
B(\beta)=1-\frac{1}{3} \frac{\left\langle\sigma^{4}\right\rangle}{\left\langle\sigma^{2}\right\rangle^{2}}
$$

and it is seen that the data are consistent with a transition which is second order or higher. We feel there is no reason to believe that the transition should be of higher than second order, since in this phase of the geometrical system the effective Hausdorff dimension is quite high which should favour mean-field results. In the phase with elongated geometry the situation is quite different. The magnetization

curve well inside this phase is shown in fig.3. There is only a gradual cross over to $|\sigma| \approx 1$ for large $\beta$, and the cross over weakens (slightly) with increasing volume. This is in agreement with the measurements of the Hausdorff dimension, $d_{H}$, in this phase which seems to indicate that $d_{H}<2$. 
The phase diagram in the $\left(\kappa_{2}, \beta\right)$ plane is as it appears for a system consisting of $9 \mathrm{~K}$ simplexes is shown in fig.4. It is in qualitative agreement with the phase diagram of $3 \mathrm{~d}$ simplicial gravity coupled to Ising spins [19]. The shaded area reflects the uncertainty in the location of the transition line separating the two phases of the geometrical system. This uncertainty is due to a discrepancy between the results for $\kappa_{2}^{c}$ arising when one uses different indicators for the change in geometry. One possible indicator is the Hausdorff dimension, $d_{H}$, another one the correlator $\left\langle R^{2}\right\rangle-\langle R\rangle^{2}$. The left boundary of the shaded area results from determining $\kappa_{2}^{c}$ as the value of $\kappa_{2}$ at the peak of $\left\langle R^{2}\right\rangle-\langle R\rangle^{2}$. The right boundary appears when $\kappa_{2}^{c}$ is defined as the value of $\kappa_{2}$ for which there is a sudden change in Hausdorff dimension. While the left hand boundary is relatively easy to determine (Cf. figure 7) the right boundary is difficult to locate precisely due to large fluctuations in geometry and should only be taken as a rough estimate. The fact that the two boundaries do not coincide for the size of systems used here should be taken as a clear sign of finite size effects. A related phenomenon is seen in the numerical studies of $2 \mathrm{~d}$ gravity coupled to Ising spins, where the peak in the specific heat does not coincide with the peak in the susceptibility due to finite size effects which seem to disappear only very slowly when the size of the system is increased. The lines of phase transition (treating the shaded area as a "line", which we expect it will be in the infinite volume limit) divide the coupling constant plane into three regions: The one to the right is characterized by no magnetization and elongated geometry, the lower left region is characterized by no magnetization and highly connected geometry, while the upper left corresponds to a magnetized phase and highly connected geometry. It is difficult to determine the exact position of the bifurcation point since we have here both a fluctuating geometry and large spin fluctuations. It is easy to understand that the transition line separating different geometries will approach the value of $\kappa_{2}^{c}$ for pure gravity when $\beta \rightarrow \infty$ and $\beta \rightarrow 0$. In these limits the spin fluctuations decouple from gravity and the locations of the transition must agree with the one of pure $4 \mathrm{~d}$ simplicial gravity.

In figure 5 we have shown the behaviour of the average curvature of our manifolds when we fix $\kappa_{2}$ inside the highly connected phase fix and move vertically in the coupling constant plane varying $\beta$. The value of $\kappa_{2}$ is the same as in figure 1 and figure 2. The position of the peak in the average curvature exactly coincides with the value of $\beta_{c}$ determined from the magnetization curve and the plot of Binders cumulant. This observation allows an easy and not so time consuming determination of $\beta_{c}\left(\kappa_{2}\right)$. The the transition line $\beta=\beta_{c}\left(\kappa_{2}\right)$ was determined using this idea. We note that this line shows little dependence on $\kappa_{2}$. The dependence of $\kappa_{2}^{c}(\beta)$ is more 
pronounced. The value of $\kappa_{2}^{c}$ is smaller for the coupled system than for pure gravity. The shift in $\kappa_{2}^{c}$ is largest when $\beta=\beta_{c}$ showing that the coupling between geometry and spins is indeed largest when the spin system is critical. This is in agreement with the intuition we have from the exactly solvable $2 \mathrm{~d}$ Ising-gravity system. The transition line $\kappa_{2}=\kappa_{2}^{c}(\beta)$ shows that effectively the spin system pushes geometry towards larger $\kappa_{2}$ values. The effect is strongest when $\beta$ is close to $\beta_{c}\left(\kappa_{2}\right)$. On the other hand we know that for large $\kappa_{2}$ values the geometry is such that the system cannot be critical. This apparent contradiction seems to be generic for the interaction between gravity and matter of the kind considered here. This is highlighted in a recent paper on multiple spin systems coupled to 2 d gravity [32]. In $2 \mathrm{~d}$ the back-reaction of the spin system on gravity is also largest close to criticality, but is such that it counteracts its own criticality by trying to deform the geometry into generic shapes where it cannot be critical (polymer-like geometries). It seems that we are observing a similar phenomenon here in $4 \mathrm{~d}$.

It is of course an interesting question whether the coupling between the spins and gravity changes the critical exponent of either of the systems as is the case in 2 dimensions. However, since the critical exponents of the pure $4 \mathrm{~d}$ gravity system are yet not known and since it has proven quite difficult to extract by numerical methods the critical exponents of the Ising spins coupled to $2 \mathrm{~d}$ gravity, we have chosen here the more modest approach to look at the influence of the spin system on bulk geometric quantities like the average curvature. As explained in the introduction this has special interest in relation to the scaling of gravity observables at the transition between geometries. We will return to this aspect after we have discussed briefly $4 \mathrm{~d}$ gravity coupled to Gaussian fields.

\subsection{Gaussian fields coupled to gravity}

In the case of Gaussian fields we have, as explained above, no coupling constant to adjust. The fields will automatically be critical in the infinite volume limit. We have considered up to four Gaussian fields coupled simultaneously to gravity and for these systems we can make a statement similar to the one made for the Ising model: The two phases of geometry seem to survive the coupling to Gaussian matter. In fig. 6 we have shown the expectation value $\left\langle\phi^{2}\right\rangle$ of a single component of the Gaussian field as a function of $\kappa_{2}$. We see a change in $\left\langle\phi^{2}\right\rangle$ linked to the change in geometry. The value of $\left\langle\phi^{2}\right\rangle$ increases when we enter into the elongated phase. In fact $\left\langle\phi^{2}\right\rangle$ also has quite large fluctuations in this phase. 


\subsection{Behaviour of gravity observables coupled to matter}

In the computer simulations we can clearly see the back-reaction of matter on the geometry for a given choice of coupling constants. It is less obvious, however, that this back reaction of matter leads to anything but trivial changes. Both for the coupling of Ising spins and Gaussian fields we still have two phases of the geometry: the highly connected one and the very elongated one. As mentioned in the introduction one could hope that the inclusion of matter would improve the scaling of the curvature at the transition. We have investigated this in the following way: As remarked above there are several indicators of the change in geometry. They result in slightly different values of $\kappa_{2}^{c}$. We have chosen here to use the peak of $\left\langle R^{2}\right\rangle-\langle R\rangle^{2}$ as an indicator of the transition, mainly because it is easier to identify than the change in Hausdorff dimension. The value of $\kappa_{2}^{c}$ depends on the matter content as can be seen from figure 7 . In fig. 8 we have plotted the average curvature as a function of the distance $\Delta \kappa_{2}$ from $\kappa_{2}^{c}$. It is seen that there is no improvement in the scaling behaviour of $\langle R\rangle\left(\kappa_{2}^{c}\right)$ as a function of the matter content, when we compare with the situation in pure gravity. In fact the curves look remarkably insensitive to the inclusion of matter and one could at this point wonder whether the back-reaction of matter has any effect on the geometry except to introduce an effective $\kappa_{2}$ which differs from the bare parameter. This is of course enough to explain the peak in the average curvature observed in figure 5 and it also provides us with an explanation why the peak is more narrow for a $9 \mathrm{~K}$ system than for a $4 \mathrm{~K}$ system. This is due to the fact that the change in average curvature across the phase transition is more sudden for the larger system. In fig. 7 we have shown $\left\langle R^{2}\right\rangle-\langle R\rangle^{2}$ for various matter fields coupled to gravity. We see that the peak grows with the number of Gaussian fields, indicating at least somewhat increased back-reaction with the number of fields. Furthermore we note that the larger the number of Gaussian fields is, the more $\kappa_{2}^{c}$ is shifted towards smaller values. Hence systems with a large number of Gaussian fields favour elongated geometries. The same phenomenon is known from two dimensions where analytic considerations show that the path integral is dominated by elongated geometries when $n_{g}$ is large. However, there is no indication that the presence of matter fields changes the nature of the phase transition of the geometrical system.

Let us comment here on a somewhat surprising feature of 4D simplicial gravity. As mentioned earlier the method of grand canonical simulation requires a finetuning

of $\kappa_{4}$ to its critical value, $\kappa_{4}^{c}$. It appears that $\kappa_{4}^{c}$ depends on $\kappa_{2}$ in a universal way. In figure 9 we have shown $\kappa_{4}^{c}\left(\kappa_{2}\right)$ for pure gravity, gravity coupled to Ising spins at 
$\beta=\beta_{c}$ and gravity coupled to 1 and 4 Gaussian fields respectively. In reference [10] 4D simplicial gravity was simulated using the following action

$$
S=\kappa_{4} N_{4}-\kappa_{2} N_{2}+\frac{h}{c_{4}^{2}} \sum_{n_{2}} o\left(n_{2}\right)\left(\frac{c_{4}-o\left(n_{2}\right)}{o\left(n_{2}\right)}\right)^{2}
$$

This corresponds to adding to the Einstein Hilbert action a typical higher derivative term (Cf. equation (田). We have shown also $\kappa_{4}^{c}\left(\kappa_{2}\right)$ for this model when $h=10$ and $h=20$. For all the systems studied $\kappa_{4}^{c}\left(\kappa_{2}\right)$ is a linear function with a slope of approximately 2.5 .

\section{Discussion}

It is clear that the numerical exploration of simplicial quantum gravity is still in its infancy. Finite size effects are not under control and it would be most desirable to be able to simulate larger systems. In principle it is possible and it will be possible in the future. But even on the small lattices used here one might reveal interesting aspects of the interaction between gravity and matter. Until now we have only considered the simplest matter systems, spins and Gaussian fields, but nothing prevents us from considering the coupling to for instance non-abelian gauge fields. It is also in principle possible to to define non-local observables like spin-spin correlation functions as functions of geodesic distance (see i.e. [19] for a discussion in the case of $3 \mathrm{~d}$ gravity) and explore their quantum averages. In this paper we have not tried to extract any critical exponents of such observables since the experience from $3 d$ is that it is not easy, and we decided in this first investigation to concentrate on bulk quantities.

The main result of the simulations is that coupling of matter to discretized gravity seems not to influence the geometry in a profound way. Of course it is possible that critical indices change (as is the case in $2 \mathrm{~d}$ gravity). Our measurements are still too poor to measure such subleading effects. As mentioned above an interesting effect would be an improved scaling of the average curvature in the region where there is a transition in geometry. We have not seen any such effect. The tentative conclusion from these first numerical experiments is that matter fields (at least of the kind we have considered here) will not add very much to our attempts to understand the basic structure of four-dimensional quantum gravity. 


\section{References}

[1] M.E. Agishtein and A.A. Migdal, Mod. Phys. Lett. A7 (1992) 1039.

[2] J. Ambjørn and J. Jurkiewicz, Phys.Lett B278 (1992) 42.

[3] H. Hamber, Nucl.Phys.B (Proc.Suppl.) 25A (1992) 150-175; H. Hamber, Phases of simplicial quantum theory in four dimensions, UCI-Th-92-29.

[4] F. David, Nucl. Phys. B 257 (1985) 45.

[5] J. Ambjørn, B. Durhuus and J. Fröhlich, Nucl. Phys. B 257 (1985) 433.

[6] F. David, Nucl. Phys. B257 (1985) 543.

[7] V. A. Kazakov, I. K. Kostov and A. A. Migdal, Phys. Lett. 157B (1985) 295.

[8] S. Varsted,UCSD-PhTh-92-03.

[9] M.E. Agishtein and A.A. Migdal, Nucl. Phys. B385 (1992) 395.

[10] J. Ambjørn, J. Jurkiewicz and C.F. Kristjansen, NBI-HE-92-53 (to appear in Nucl. Phys. B).

[11] B. Brügmann, Non-uniform Measure in Four-Dimensional simplicial Quamtum Gravity, SU-GP-92/9-1.

[12] B. Brügmann and E. Marinari, 4d Simplicial Quantum Gravity with a NonTrivial Measure, SU-GP-92/9-2.

[13] I. Antoniadis, P.O. Mazur and E. Mottola, Scaling behavior of quantum fourgeometries, Preprint CPTH-A214.1292

[14] V. Knizhnik, A. Polyakov and A. Zamolodchikov, Mod. Phys. Lett. A3 (1988) 819.

[15] V.A. Kazakov, Phys. Lett 119A (1986) 140.

[16] D. Boulatov and V.A. Kazakov, Phys. Lett. 184B (1987) 247.

[17] C. Baillie, Phys. Rev. D46, (1992) 2480.

[18] R. Renken, S. Catterall and J. Kogut, ILL-TH-92-7.

[19] J. Ambjørn, Z. Burda, J. Jurkiewicz and C.F. Kristjansen, Phys. Lett 297B (1992) 253.

[20] J. Ambjørn, D. Boulatov, A. Krzywicki and S. Varsted, Phys.Lett B276 (1992) 432.

[21] J. Ambjørn and S. Varsted, Nucl.Phys. B373 (1992) 557. 
[22] J. Ambjørn, B. Durhuus, J. Fröhlich and P. Orland, Nucl.Phys. B270 (1986) 457.

[23] J. Ambjørn, B. Durhuus, J. Fröhlich, Nucl. Phys. B275 (1986) 161.

[24] D. Boulatov, V. A. Kazakov, I. K. Kostov and A. A. Migdal, Phys.Lett. 174B (1986) 87.

[25] J. Jurkiewicz, A. Krzywicki and B. Petersson, Phys.Lett. 168B (1986) 273; 177B (1986) 89.

[26] J. Ambjørn, Ph. De Forcrand, F. Koukiou and D. Petritis, Phys.Lett 197B (1987) 548.

[27] U. Wolff, Phys.Rev.Lett. 62 (1989) 361.

[28] Z.Burda, J.Jurkiewicz and L.Kärkkäinen, Comput. Phys. Commun. 70 (1992) 510 .

[29] C.F. Baillie and D.A. Johnston, Phys. Lett. B286 (1992) 44;

[30] C.F. Baillie and D.A. Johnston, Mod. Phys. Lett. A7 (1992) 1519.

[31] S. M. Catterall, J.B. Kogut and R.L. Renken, Phys. Lett B292 (1992) 277; Phys. Rev. D45 (1992) 2957.

[32] J. Ambjørn, B. Durhuus, T. Jonsson and G. Thorleifsson, NBI-HE-92-35 (to appear in Nucl. Phys. B) 


\section{Figure Captions}

Fig. 1 The absolute value of the magnetization, as defined by (11), as a function of $\beta$ for $\kappa_{2}=0.9$, i.e. in the phase with a highly connected geometry. The circles correspond to a volume $N_{4}=4000$, the triangles to $N_{4}=9000$.

Fig. 2 Binder's cumulant (12) for $\kappa_{2}=0.9$ and three volumes: $N_{4}=4000(\nabla)$, $N_{4}=6000(\square)$ and $N_{4}=9000(\bigcirc)$. The shape corresponds to a transition of second or higher order and the point of intersection to $\beta_{c}\left(N_{4}=\infty\right)$.

Fig. 3 The absolute value of the magnetization, as defined by (11), as a function of $\beta$ for $\kappa_{2}=1.3$, i.e. in the phase with elongated geometry. The circles correspond to a volume $N_{4}=4000$, the triangles to $N_{4}=9000$.

Fig. 4 The phase diagram in the $\left(\kappa_{2}, \beta\right)$ plane as it appears when $N_{4}=9000$. As discussed in the text there are reasons to believe that part of the diagram is distorted by finite size effects and that the in the infinite volume the shaded region will be replaced by the dashed line.

Fig. 5 The effect on the curvature $\langle R\rangle-\langle R\rangle_{0}$ (where $\langle R\rangle$ is defined by (3)) when we are in the phase with a large Hausdorff dimension and change $\beta$. The value of $\kappa_{2}=0.9$ and the circles correspond to $N_{4}=4000$ while the triangles correspond to $N_{4}=9000$. $\langle R\rangle_{0}$ denotes the average curvature in the case of pure gravity (it differs slightly for $N_{4}=4000$ and $N_{4}=9000$ due to finite size effects).

Fig. 6 The change in $\left\langle\phi^{2}\right\rangle$ (a single component field) as a function of $\kappa_{2}$ for $N_{4}=4000$.

Fig. $7\left\langle R^{2}\right\rangle-\langle R\rangle^{2}$ for a different matter fields as a function of $\Delta \kappa_{2}$. Pure gravity $(\nabla)$, gravity + Ising at $\beta_{c},(+)$, gravity +1 Gaussian field $(\bigcirc)$ and gravity +4 Gaussian fields $(\square)$. (The observables $\langle R\rangle$ and $\left\langle R^{2}\right\rangle$ are defined in (3) and (4) respectively.)

Fig. $8\langle R\rangle$ as a function of $\Delta \kappa_{2}$ for different matter content. Pure gravity $(\nabla)$, gravity + Ising at $\beta_{c},(+)$, gravity +1 Gaussian field $(\bigcirc)$ and gravity +4 Gaussian fields $(\square)$.

Fig. $9 \kappa_{4}^{c}$ as a function of $\kappa_{2}$ for different systems. Pure gravity $(\times)$, gravity + Ising at $\beta=\beta_{c}(\bigcirc)$, gravity +1 Gaussian field $(\square)$, gravity +4 Gaussian fields $(\triangle)$, gravity with higher derivative term for $h=10(\bullet)$ and gravity with higher derivative term for $h=20(+)$. 